\title{
ZTF J1901+5309: a 40.6-min orbital period eclipsing double white dwarf system
}

\author{
Michael W. Coughlin ${ }^{\oplus}, 1,2 \star$ Kevin Burdge ${ }^{2}$ E. Sterl Phinney, ${ }^{2}$ Jan van Roestel, ${ }^{2}$ \\ Eric C. Bellm, ${ }^{3}$ Richard G. Dekany, ${ }^{4}$ Alexandre Delacroix,${ }^{4}$ Dmitry A. Duev ${ }^{\bullet},{ }^{2}$ \\ Michael Feeney, ${ }^{4}$ Matthew J. Graham ${ }^{\oplus},{ }^{2}$ S. R. Kulkarni, ${ }^{2}$ Thomas Kupfer, ${ }^{5}$ \\ Russ R. Laher, ${ }^{6}$ Frank J. Masci, ${ }^{6}$ Thomas A. Prince ${ }^{\odot}, 2$ Reed Riddle ${ }^{\odot}, 4$ \\ Philippe Rosnet, ${ }^{7}$ Roger Smith, ${ }^{4}$ Eugene Serabyn ${ }^{8}$ and Richard Walters ${ }^{4}$ \\ ${ }^{1}$ School of Physics and Astronomy, University of Minnesota, Minneapolis, MN 55455, USA \\ ${ }^{2}$ Division of Physics, Math, and Astronomy, California Institute of Technology, Pasadena, CA 91125, USA \\ ${ }^{3}$ DIRAC Institute, Department of Astronomy, University of Washington, 3910 15th Avenue NE, Seattle, WA 98195, USA \\ ${ }^{4}$ Caltech Optical Observatories, California Institute of Technology, Pasadena, CA 91125, USA \\ ${ }^{5}$ Kavli Institute for Theoretical Physics, University of California, Santa Barbara, CA 93106, USA \\ ${ }^{6} I P A C$, California Institute of Technology, 1200 E. California Blvd, Pasadena, CA 91125, USA \\ ${ }^{7}$ Université Clermont Auvergne, CNRS/IN2P3, LPC, Clermont-Ferrand 63001, France \\ ${ }^{8}$ Jet Propulsion Laboratory, California Institute of Technology, Pasadena, CA 91109, USA
}

Accepted 2020 March 6. Received 2020 March 5; in original form 2020 February 17

\begin{abstract}
The Zwicky Transient Facility has begun to discover binary systems with orbital periods that are less than $1 \mathrm{~h}$. Combined with dedicated follow-up systems, which allow for high-cadence photometry of these sources, systematic confirmation and characterization of these sources are now possible. Here, we report the discovery of ZTF J190125.42+530929.5, a 40.6-min orbital period, eclipsing double white dwarf binary. Both photometric modelling and spectroscopic modelling confirm its nature, yielding an estimated inclination of $i=86.2_{-0.2}^{+0.6} \mathrm{deg}$ and primary and secondary effective temperatures of $T_{\text {eff }}=28000_{-500}^{+500}$ and $17600_{-400}^{+400} \mathrm{~K}$, respectively. This system adds to a growing list of sources for future gravitational-wave detectors and contributes to the demographic analysis of double degenerates.
\end{abstract}

Key words: (stars:) binaries: eclipsing -(stars:) white dwarfs.

\section{INTRODUCTION}

The data from the current generation of astronomical surveys, with a variety of cadences, sensitivities, and fields of view, are resulting in an incredible variety of time-scales to study variable stars. These surveys include the Panoramic Survey Telescope and Rapid Response System (Pan-STARRS; Morgan et al. 2012), the Optical Gravitational Lensing Experiment (Soszyński et al. 2013), the AllSky Automated Survey for Supernovae (Jayasinghe et al. 2018), the Asteroid Terrestrial-impact Last Alert System (Tonry et al. 2018), and the Zwicky Transient Facility [ZTF; Bellm et al. (2018), Graham et al. (2019), and Masci et al. (2018)] among others. The study of variable stars is an important part of understanding stellar and galactic structure and evolution. For example, some classes of variable stars such as Cepheids and RR Lyrae stars are standard candles and intrinsically bright, making them useful tracers of

^E-mail: cough052@umn.edu galactic structure (Skowron et al. 2019). Due to the cadence at which many previous surveys have returned to the same location on the sky $(\geq 1 \mathrm{~d}$ ), relatively little is known about the short-time-scale variable optical sky. Below this nominal cadence, repeated observations of the same field at different phases of potential periodic sources allow for potential identification of variability at much shorter time-scales.

Confirmation and characterization of the shortest period variables require a follow-up by dedicated resources. For example, the Kitt Peak Electron Multiplying CCD (EMCCD) demonstrator (KPED), which is an EMCCD and filter wheel combination on the Kitt Peak 84-inch telescope, is designed for rapid and sensitive photometric follow-up of these sources (Coughlin et al. 2019). A combination of its low-noise characteristics $\left(<1 e^{-} \mathrm{rms}\right)$, rapid readout speeds $(\approx 8 \mathrm{~Hz})$, and field of view $\left(4.4 \times 4.4 \mathrm{arcmin}^{2}\right.$ field of view $)$ is able to resolve low-amplitude variability on time-scales of seconds.

One example of potentially short-period, periodic sources is white dwarf (WD) stars, which arise from the final stage of stellar evolution for stars with initial masses below approximately 7-9 $\mathrm{M}_{\odot}$ (Dobbie et al. 2006). Future gravitational-wave detector missions 
in the millihertz band will detect WD binary systems; short-period and non-interacting binary systems make for excellent verification sources for these detectors (Kupfer et al. 2018) because it is possible to monitor the orbital decay in the optical for comparison to general relativity and the data from these gravitational-wave observatories. Some of the shortest period examples of this class are the 12.7min system SDSS J065133.338+284423.37 (known as J0651) (Brown et al. 2011; Hermes et al. 2012) and the 6.9-min system ZTF J153932.16+502738.8 (known as ZTF J1539+5027) (Burdge et al. 2019a). Many of these systems will merge within a Hubble time due to the loss of angular momentum through gravitationalwave radiation. Detached systems in particular are important, as interacting systems complicate direct tests of general relativity.

In this work, we highlight a 40.6-min period, WD binary system, ZTF J1901+5309, discovered by ZTF, with the eclipses and period confirmed by KPED. The system most similar to the one presented here is SDSSJ010657.39-100003.3 (known as J0106-1000), which is a detached WD system with an orbital period of only $39.1 \mathrm{~min}$, and was the first detection of a tidally distorted WD (Kilic et al. 2011). ZTF J1901+5309 has magnitudes of $g=17.9 \mathrm{mag}, r=18.3 \mathrm{mag}$, $i=18.6 \mathrm{mag}$, and $N U V=17.3 \mathrm{AB}$ mag (from Galex, Bianchi, Shiao \& Thilker 2017), and from Gaia (Gaia Collaboration 2018), a parallax $=1.1 \pm 0.1$ mas corresponding to $D=900_{-90}^{+110} \mathrm{pc}$. It has a right ascension of $19 \mathrm{~h} 01 \mathrm{~m} 25.42 \mathrm{~s}$ and declination of $53^{\circ} 09^{\prime}$ $29.5^{\prime}$ (epoch J2000). Section 2 gives the selection criteria used to identify variable ZTF sources. Section 4 presents the observations for this system. We present our conclusions and paths for future work in Section 5.

\section{SELECTION CRITERIA}

We discovered ZTF J1901+5309 by running the conditional entropy period-finding algorithm (Graham et al. 2013) over the ZTF observations of a sample of all Pan-STARRS objects satisfying the colour cut $g-r<0.0$ and $r-i<0.0$, in the same manner as described in Burdge et al. (2019a). We selected a subset of these objects with more than 50 available ZTF epochs at the time, and combined the $g$, $r$, and $i$-band light curves by matching the median brightness values in each band. We required a minimum of $50 \mathrm{ZTF}$ epochs in these light curves, which resulted in a sample of $\approx 10$ million objects. We phase folded objects at their 'best' period (which corresponds to the minimum entropy), and saved thumbnails of each phase folded light curve. Then, by manual inspection, we selected systems to follow up (in particular, those that appeared to have a string of significant negative outliers phasing up into an eclipse-like feature). ZTF J1901+5309 was the second such object we identified after ZTF J1539+5027 (Burdge et al. 2019a). This was possible due to its location in the ZTF high declination survey, which has a sampling cadence of 6 times per night ( 3 in the ZTF $g$ band and 3 in the ZTF $r$ band); this is the same survey in which ZTF J1539+5027 was discovered. As of the time of publication, ZTF J1901+5309 is consistently recovered as one of the highest significance periodic objects with a period under an hour using conditional entropy, and in searches using the box least squares algorithm (Kovács, Zucker \& Mazeh 2002), is the most significant periodic object we detect out of all WD candidates identified in the Gentillo-Fusillo WD catalogue (Gentile Fusillo et al. 2019).

\section{OBSERVATIONS}

On the left of Fig. 1, we show the ZTF light curve available around the time of identification, phase folding at the highest likelihood period (see Table 1), plotting points at the mid-exposure time of ZTF's 30 s exposures. As of ZTF's second data release, ${ }^{1}$ there are 845 observations in the ZTF $g$ band, 928 observations in the ZTF $r$ band, and 62 observations in the ZTF $i$ band. The right of Fig. 1 shows the KPED light curve, confirming the object as an eclipsing binary. Circular, fixed-aperture photometry is performed on the KPED and CHIMERA images (Coughlin et al. 2019). Differential photometry from a bright, nearby comparison star is used to remove transparency variations. Differential atmospheric extinction resulting in long-term photometric trends is removed using a loworder polynomial. All observations are converted from modified Julian date in coordinated universal time (UTC) to barycentric Julian date in barycentric dynamical time (TDB) using a light-traveltime correction in astropy (Astropy Collaboration 2018) to account for the motion of the Earth around the barycentre of the Solar system.

Our spectroscopic observations are from the DoubleSpec (DBSP) spectrograph of the Hale 200-inch telescope at Palomar Observatory on the night of 2019-05-29. We obtained 8 epochs of spectroscopy of the system. Using the $600 / 4000$ blue grating with an angle at $27 \mathrm{deg}$ 17 min and a 1.5-arcmin slit resulted in $1 \AA$ resolution and coverage from 3400 to $5700 \AA$. At this resolution, exposures of 360 s were possible, shown in Fig. 5. We also obtained 72180 s exposures on the night of 2019-09-03 using the low-resolution imaging spectrometer (LRIS) (Oke et al. 1995) on the 10-m W. M. Keck I Telescope on Mauna Kea, using the 400/8500 grism and $2 \times 2$ binning on the blue arm. The signal-to-noise ratio in each observation is $\sim 10$, with wavelength coverage from 3200 to $9500 \AA$, and a resolution of approximately $\Delta \lambda / \lambda=3000$.

\section{RESULTS}

A period search of the ZTF light curve shows an orbital period of $P=40.6 \mathrm{~min}$. We do not see significant ellipsoidal variations, which indicates that the brighter object is not being significantly distorted due to rotation and tides, and thus is well within its Roche lobe. We use the $g$ band CHIMERA light curve in Fig. 2 to perform the light-curve modelling. Following the method from Burdge et al. (2019a), we use a python implementation of Mult inest (Feroz, Hobson \& Bridges 2009) to measure the radial-velocity covariances. The sample light curves were generated using the ellc package (Maxted 2016), where the light curves depend on the mid-eclipse time of the primary eclipse, $t_{0}$, the inclination, $i$, the mass ratio, $q=\frac{m_{2}}{m_{1}}$, the ratio of the radii to the semimajor axis, $r_{1}=R_{1} / a$, $r_{2}=R_{2} / a$, and the surface brightness ratio, $J$. The first object is treated as spherical, and the second is assumed to have a Roche geometry. We use the period derived from the ZTF observations. The resulting parameters from this fit are given in Table 1. We note that the mass ratio in the light-curve modelling is very poorly constrained due to the lack of ellipsoidal modulation present. There are some signs of one object being irradiated by the other; this effect can be useful in constraining the surface brightness ratio in such systems. However, in an edge-on system with two eclipses such as this one, this quantity is much more strongly constrained by the relative depths of the eclipses.

Since the spectra (Fig. 3) show broad Balmer lines of hydrogen from both WDs, we use a library of synthetic spectra for WDs with hydrogen-rich (DA) atmospheres from Levenhagen et al. (2017) to estimate the temperature and surface gravity. The spectral grid covers the range $17000 \mathrm{~K} \leq T_{\text {eff }} \leq 100000 \mathrm{~K}$ and the surface gravity

\footnotetext{
${ }^{1}$ https://www.ztf.caltech.edu/page/dr2
} 

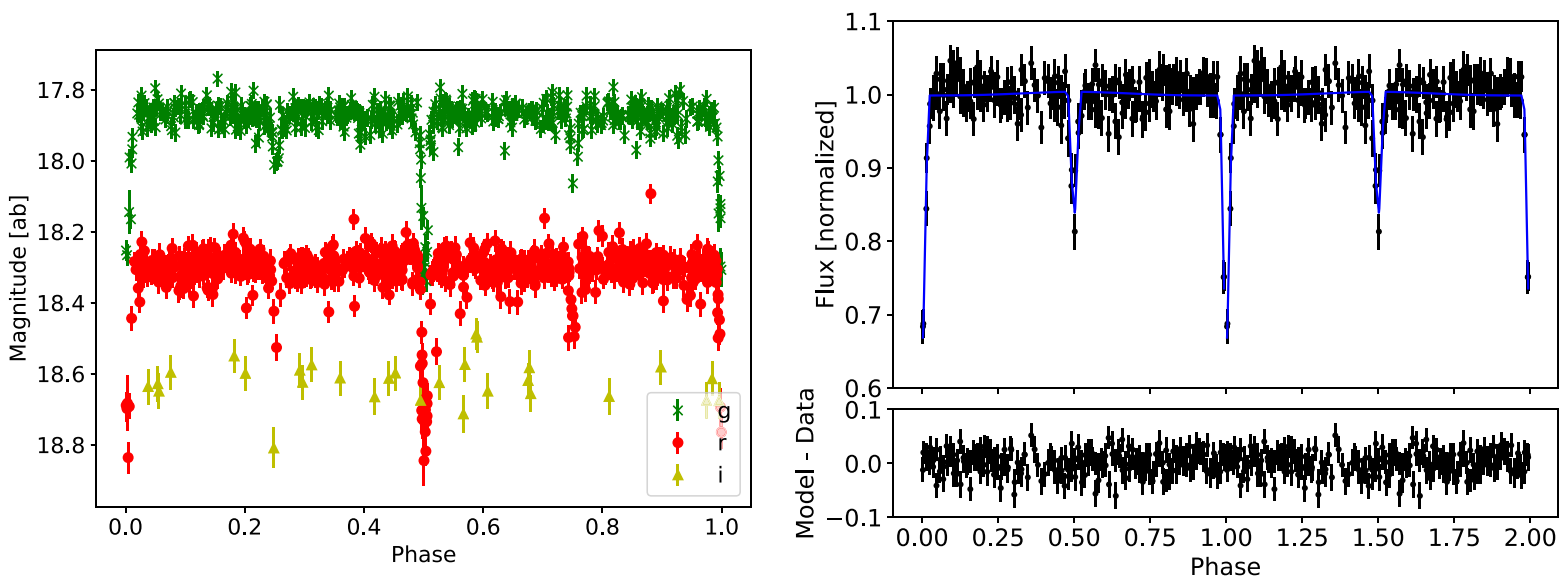

Figure 1. On the left are the ZTF composite light curves of ZTF J190125.42+530929.5 (hereafter ZTF J1901+5309) with data taken between 2018 March and July (the approximate amount of data used for the identification step). On the right is the confirmation photometry from KPED observations in $g^{\prime}$, taken on the nights of 2019-03-04, 2019-03-18, 2019-10-03, and 2019-11-16; these sets of $1 \mathrm{~s}$ duration observations lasted $\sim 2 \mathrm{~h}$ each. The blue trace is the best-fitting model.

Table 1. Table of physical parameters. $a$ is the orbital separation, $J$ is the surface brightness ratio of secondary to the primary, and $g$, the surface gravity, is in cgs units. The astrometry (epoch J2000), parallax, and distance measurements are taken from Gaia (Bailer-Jones et al. 2018; Gaia Collaboration 2018)

\begin{tabular}{lc}
\hline$R_{\mathrm{A}}$ & $0.07_{-0.01}^{+0.01} a$ \\
\hline$R_{\mathrm{B}}$ & $0.08_{-0.02}^{+0.01} a$ \\
$J$ & $0.42_{-0.02}^{+0.02}$ \\
$i$ & $86.2_{-0.2}^{+0.6} \mathrm{deg}$ \\
$T_{0}$ & $2458703.87381_{-0.00001}^{+0.0001} \mathrm{BJD}_{\mathrm{TDB}}$ \\
$q$ & $0.21_{-0.15}^{+0.53}$ \\
$T_{\mathrm{A}}$ & $28000_{-500}^{+500} \mathrm{~K}$ \\
$T_{\mathrm{B}}$ & $17600_{-400}^{+400} \mathrm{~K}$ \\
$\log (g)_{\mathrm{A}}$ & $7.6_{-0.3}^{+0.3}$ \\
$P\left(T_{0}\right)$ & $2436.11_{-0.03}^{+0.02} \mathrm{~s}$ \\
$\mathrm{RA}$ & \\
& \\
Dec & $285.35592397 \pm 0.00000003 \mathrm{deg}$ \\
Parallax & $1.1 \pm 0.1 \mathrm{mas}$ \\
$D$ & $915_{-80}^{+96} \mathrm{pc}$ \\
\hline
\end{tabular}

range $7.0 \leq \log (g) \leq 9.5$. While the models only span a certain range of $\log (g)$ and helium abundance, as we observe DA WDs, they should be reasonable to use. We use a modification of a previously published, Gaussian Process Regression-based technique (Coughlin et al. 2018) to create surrogate spectral models to interpolate the spectra across the parameter space of interest. We de-redden all of the spectra using the model of Fitzpatrick \& Massa (2007). Taking the spectrum nearest an orbital phase $\sim 0$, we find that the primary has $T_{\text {eff }}=28000 \pm 500$ and $\log (g)=7.6 \pm 0.3$ in Fig. 3 . We find similar measurements across the rest of the DBSP as well as the LRIS spectra (see Fig. 4). This implies a likely helium $\mathrm{WD}$, given its relatively low $\log (g)$ and large ratio of radius to semimajor axis at this orbital period, although it could also be consistent with a low-mass carbon-oxygen core WD. Assuming both objects are approximately consistent with blackbodies in the

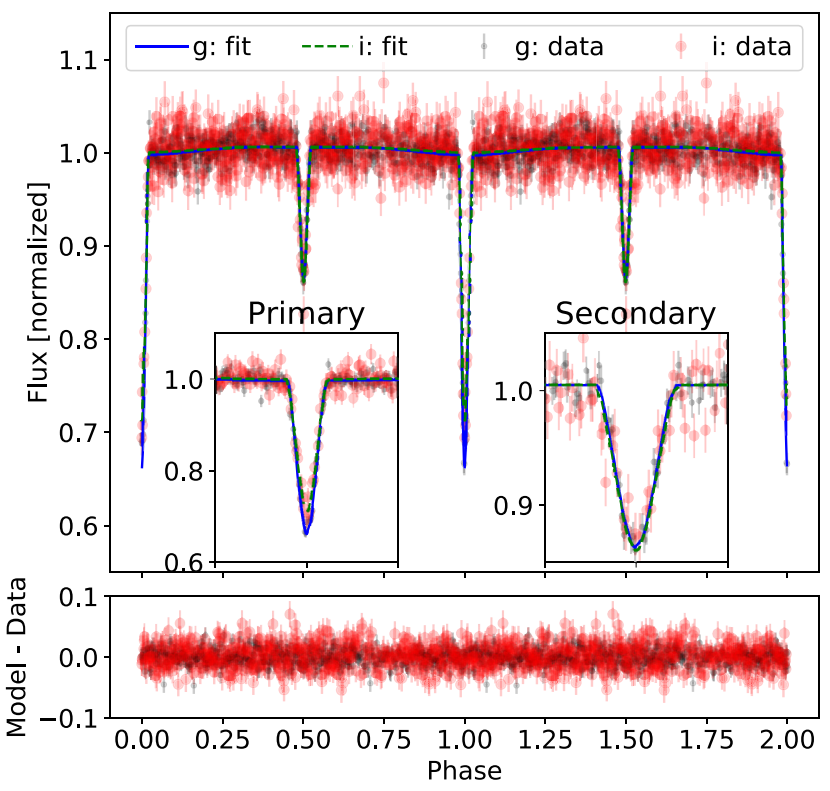

Figure 2. $g^{\prime}$ (black markers) and $i^{\prime}$ (red markers) light curves of ZTF J1901+5309 from CHIMERA (Harding et al. 2016), the high-cadence photometer on the Palomar-200 in, taken on the nights of 2019-04-29 and 2019-08-08. Here, we show $3 \mathrm{~s}$ integrations beginning August 8, 2019 8:16:46 UTC and ending August 8, 2019 10:33:52 UTC. The blue and green traces are the best-fitting models for $g^{\prime}$ and $i^{\prime}$, respectively. We include insets showing zoomed versions of the primary and secondary eclipses as well.

observed passbands, and using the surface brightness ratio derived from the light-curve modelling, we find that the secondary has a temperate of $T=17600_{-400}^{+400} \mathrm{~K}$.

We expect that these measurements may be biased by the potential contamination from the companion; we note that because the eclipse is not total, it includes a contribution from the secondary, and the overlap between the lines from the primary and secondary makes joint fits difficult. In particular, it is likely that $\log (g)$ may be overestimated. We note in particular that the cooler companion is expected to have much wider $(\sim 2 \times)$ line widths than the primary, e.g. fig. 10 of Terashita \& Matsushima (1966), and so contamination 


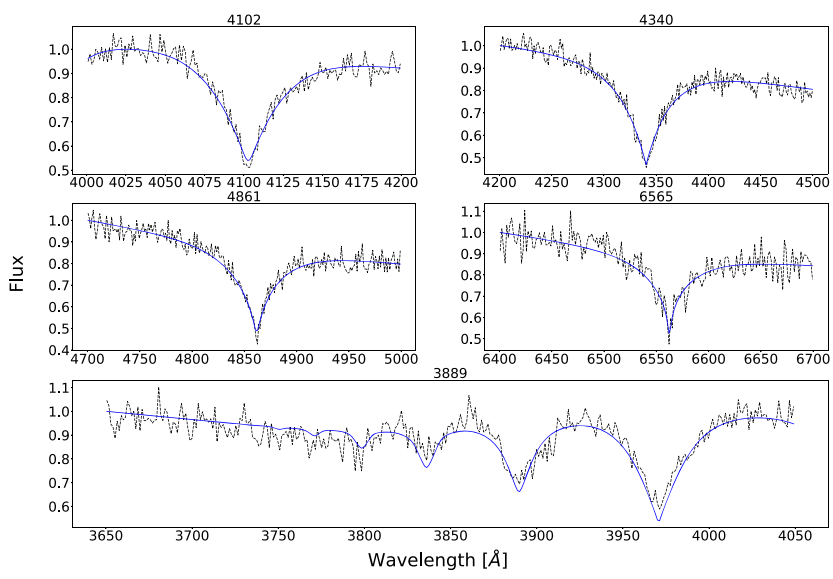

Figure 3. Fits of the surrogate model based on the simulations of Levenhagen et al. (2017) fit to the LRIS spectroscopy at an orbital phase $\sim 0$. This minimizes the artificial broadening of the lines as the WD spectra are superimposed, rather than separated. We highlight a handful of lines (the central wavelength shown in the plot titles) showing the spectral fits.

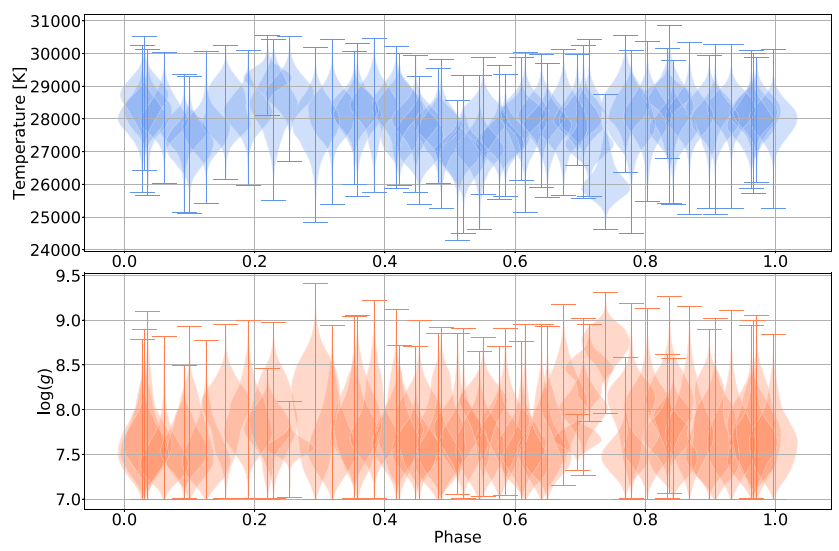

Figure 4. 'Violin plot' for the temperature and $\log g$ fits for LRIS spectra of ZTF J1901+5309 throughout its orbit.

of the wings by the companion is potentially significant. In addition, Stark broadening could be significant. Given that the pressure at a given optical depth varies roughly as $g^{2 / 3}$, e.g. fig. 1 of Terashita \& Matsushima (1966) and Stark broadening has absorption coefficient $\alpha \propto(p / T) \Delta \lambda^{-5 / 2}$, therefore, at a given optical depth in the wing, $\Delta \lambda \propto g^{4 / 15}, g \propto \Delta \lambda^{3.75}$. In this way, the $\sim 20$ per cent variations in the line widths here could therefore give a spurious increase in the apparent $g$ by a factor of 2 , or 0.3 in $\log (g)$. As a point of comparison, we can use the Gaia (Gaia Collaboration 2018) distance to make a blackbody radius estimate, with $F_{v}=R_{A}^{2} \pi B_{v}(T) / D^{2}$. Using a blackbody temperature of $28000 \mathrm{~K}$ and the extinction-corrected $g$ band flux, we find $R_{A}=1.85 \times 10^{9} \mathrm{~cm}$; it is likely that such large radii are only attainable for hot WDs of less than about $0.35 \mathrm{M}_{\odot}$ (or $\log g \lesssim 7.2$ ); see e.g. fig 3. of Panei, Althaus \& Benvenuto (2000).

Given that it is likely that the stars have already interacted in two common envelope phases, with potential mass exchange, evolutionary models do not apply here; however, single star evolutionary models, e.g. Camisassa et al. (2019) and Tremblay, Bergeron \& Gianninas (2011), could in principle be used to guide plausible ranges of radii for both $\mathrm{He}$ and $\mathrm{C} / \mathrm{O}$ WDs.

We now use the phase-resolved spectroscopy from LRIS to measure the orbital velocities of the WDs in the binary (see Burdge

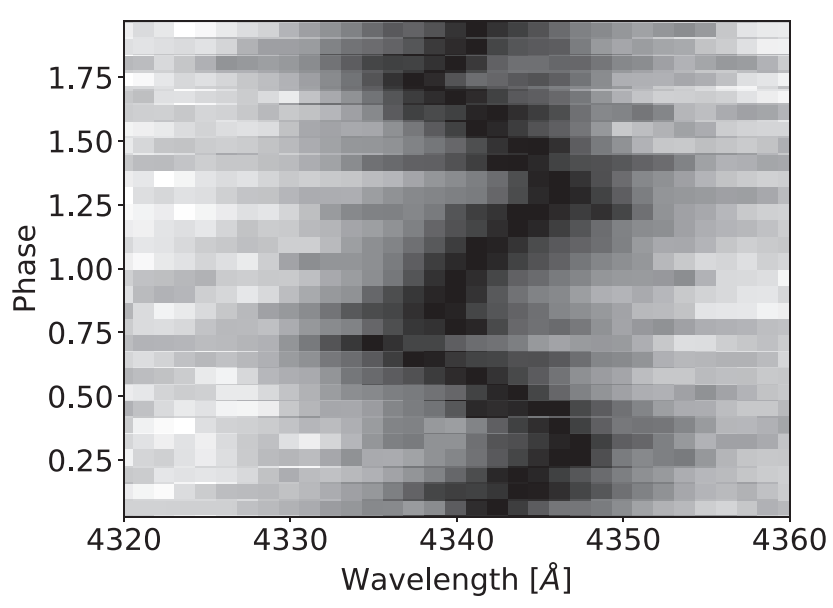

Figure 5. Array of LRIS spectra of ZTF J1901+5309 throughout its orbit focused on the $\mathrm{H} \gamma$ transition at $\approx 4340 \AA$.

et al. 2019a, for a full discussion). The spectra exhibit broad and shallow hydrogen absorption lines, which are characteristic of a hot DA WD, in Fig. 4. There are two overlapping absorption features here that move out of phase with one another; thus, this is a 'doublelined' spectroscopic binary. We once again use Mult inest (Feroz et al. 2009) to measure the radial-velocity covariances. As a simple model, we use Gaussians to fit the line associated with the $n=$ 5-2 transition of hydrogen in each spectrum. This yields a radialvelocity semi-amplitude of the objects, $K_{1}$ and $K_{2}$, which we fit to a sine wave

$v_{i}(n)=K_{\mathrm{i}} \sin \left(\frac{2 \pi n}{N}+\phi_{i}\right)+A$,

where $n$ encodes the index of the phase bin, $\phi_{1}=0$, and $\phi_{2}=$ $\pi, A$ is the systemic velocity, and $v_{i}(n)$ is the velocity associated with $\phi_{i}$. We used a one-dimensional Kernel Density Estimator for each $K_{\mathrm{i} \text {, measured }}$ to assign the probabilities during sampling. Unfortunately, due to the significant blending of the lines, the semiamplitudes are significantly underestimated at $200 \mathrm{~km} \mathrm{~s}^{-1}$. For this reason, while the changes in radial velocity are clear (see Fig. 5), it is not currently possible to extract quantitative measurements; while this does give a lower limit for comparison with future analyses, it gives an unphysically small mass function.

Given that we do not have a chirp mass, we take as a proxy a $0.5+0.3$ solar-mass WD system. With this assumption, knowledge of the orbital period, and the distance estimate from Gaia, we can estimate the gravitational-wave strain of the system. To calculate the characteristic strain (Korol et al. 2017), $S_{\mathrm{c}}$, we use

$S_{\mathrm{c}}=\frac{2\left(G M_{\mathrm{c}}\right)^{5 / 3}(\pi f)^{2 / 3}}{c^{4} D} \sqrt{f T_{\mathrm{obs}}}$,

where $c$ is the speed of light and $T_{\mathrm{obs}}$ is the operation time of the LISA mission. Marginalizing over the distance uncertainties, we provide the point estimate and uncertainties in Fig. 6, along with other known LISA sources. Accounting for the LISA's instrument response patterns (Korol et al. 2017), the LISA signal-to-noise ratio is around $2.4_{-0.3}^{+0.3}$ at the end of the nominal 4-yr mission lifetime, which would be on the edge of detectability. For a $0.5+0.3$ solar-mass WD system, gravitational radiation will bring them into contact in approximately $2 \times 10^{7} \mathrm{yr}$, when the orbital period will be around 5 min. The subsequent fate of the system after the onset of mass transfer depends sensitively on the (ill-determined) mass ratio, tidal coupling, and response to mass and angular momentum transfer 


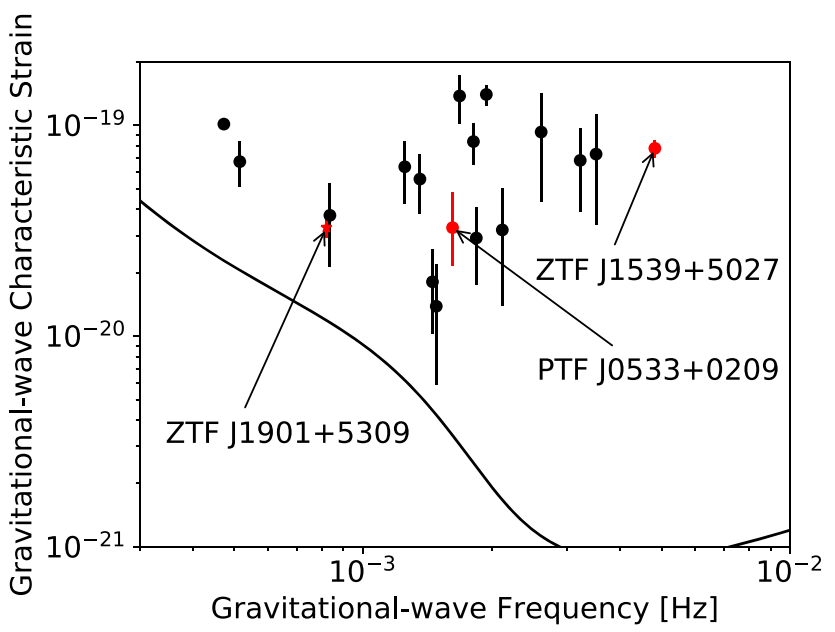

Figure 6. Known LISA detectable binaries assuming a 4-yr observation time with the LISA sensitivity curve in black. The previously known sources in black are taken from Kupfer et al. (2018), while the red star is ZTF $\mathrm{J} 1901+5309$ and the red circles indicate other sources from the PTF/ZTF search for LISA sources (Burdge et al. 2019a, b).

(Marsh, Nelemans \& Steeghs 2004; Shen 2015), and could end its life as an SN .Ia or an R Coronae Borealis star (Shen 2015).

\section{CONCLUSION}

In this work, we have described the discovery and characterization of the detached WD binary system ZTF J1901+5309, one of a growing number of systems that will be important sources for future space-based gravitational-wave detectors. We have illustrated the importance of high-cadence photometric follow-up using target selection from ZTF to enable the robust identification and confirmation of short-period variables in particular. Using photometric and spectroscopic follow-up, we have constrained the WD mass ratio and scaled radii. The discovery of these systems is important for a variety of reasons. First of all, the measurement of the space density and merger rate of WD binaries enables the test of whether they are the source of type Ia supernovae or other explosive events, e.g. fig. 3 of Shen (2015). In addition, there are a variety of predictions for the resulting evolution of sources of this type. For example, if the mass ratio is less than 0.6 , stable mass transfer could lead to the creation of AM CVn systems like HM Cancri (Roelofs et al. 2010). It is also possible that unstable mass transfer will cause the WDs to quickly coalesce and merge (Dan et al. 2011; Shen 2015).

Going forward, follow-up high-cadence photometry will play an important role in confirmation of these systems and as a timing instrument. By the time LISA flies, high-cadence follow-up will allow for the measurement of the time derivative of the orbital period with time for many systems that show eclipses and have periods that are less than $1 \mathrm{~h}$. These objects require regular monitoring over the course of months to years to measure a change in period, depending on the masses and initial period (at discovery) of the objects. There are ongoing observations to determine the orbital decay rate for this system.

In addition, this system in particular will benefit from higher resolution spectra in order to disentangle the radial velocity measurements. While a measurement of the rate of change of orbital period is possible, more straightforwardly, this would enable measurement of the system's chirp mass; without either of these measurements, it may remain unknown until LISA flies.

\section{ACKNOWLEDGEMENTS}

MC is supported by the David and Ellen Lee Prize Postdoctoral Fellowship at the California Institute of Technology. SRK thanks the Heising-Simons Foundation for supporting his research with ZTF. Part of this work was carried out at the Jet Propulsion Laboratory, under contract with NASA. ESP's research was funded in part by the Gordon and Betty Moore Foundation through Grant GBMF5076.

Based on observations obtained with the Samuel Oschin Telescope 48-inch and the 60-inch Telescope at the Palomar Observatory as part of the ZTF project. ZTF is supported by the National Science Foundation under Grant No. AST-1440341 and a collaboration including Caltech, IPAC, the Weizmann Institute for Science, the Oskar Klein Center at Stockholm University, the University of Maryland, the University of Washington (UW), Deutsches Elektronen-Synchrotron and Humboldt University, Los Alamos National Laboratories, the TANGO Consortium of Taiwan, the University of Wisconsin at Milwaukee, and Lawrence Berkeley National Laboratories. Operations are conducted by Caltech Optical Observatories, IPAC, and UW.

The KPED team thanks the National Science Foundation and the National Optical Astronomical Observatory for making the Kitt Peak 2.1-m telescope available. We thank the observatory staff at Kitt Peak for their efforts to assist Robo-AO KP operations. The KPED team thanks the National Science Foundation, the National Optical Astronomical Observatory, the Caltech Space Innovation Council, and the Murty family for support in the building and operation of KPED. In addition, they thank the CHIMERA project for use of the Electron Multiplying CCD (EMCCD).

\section{REFERENCES}

Astropy Collaboration, 2018, AJ, 156, 123

Bailer-Jones C. A. L., Rybizki J., Fouesneau M., Mantelet G., Andrae R., 2018, AJ, 156, 58

Bellm E. C. et al., 2018, PASP, 131, 018002

Bianchi L., Shiao B., Thilker D., 2017, ApJS, 230, 24

Brown W. R., Kilic M., Hermes J. J., Allende Prieto C., Kenyon S. J., Winget D. E., 2011, ApJ, 737, L23

Burdge K. B., Coughlin M. W., Fuller J., 2019a, Nature, 571, 528

Burdge K. B. et al., 2019b, ApJ, 886, L12

Camisassa M. E. et al., 2019, A\&A, 625, A87

Coughlin M. W. et al., 2018, MNRAS, 480, 3871

Coughlin M. W. et al., 2019, MNRAS, 485, 1412

Dan M., Rosswog S., Guillochon J., Ramirez-Ruiz E., 2011, ApJ, 737, 89

Dobbie P. D., Napiwotzki R., Lodieu N., Burleigh M. R., Barstow M. A., Jameson R. F., 2006, MNRAS, 373, L45

Feroz F., Hobson M. P., Bridges M., 2009, MNRAS, 398, 1601

Fitzpatrick E. L., Massa D., 2007, ApJ, 663, 320

Gaia Collaboration, 2018, A\&A, 616, A1

Gentile Fusillo N. P. et al., 2019, MNRAS, 482, 4570

Graham M. J., Drake A. J., Djorgovski S. G., Mahabal A. A., Donalek C., 2013, MNRAS, 434, 2629

Graham M. J. et al., 2019, PASP, 131, 078001

Harding L. K. et al., 2016, MNRAS, 457, 3036

Hermes J. J. et al., 2012, ApJ, 757, L21

Jayasinghe T. et al., 2018, MNRAS, 477, 3145

Kilic M. et al., 2011, MNRAS, 413, L101

Korol V., Rossi E. M., Groot P. J., Nelemans G., Toonen S., Brown A. G. A., 2017, MNRAS, 470, 1894

Kovács G., Zucker S., Mazeh T., 2002, A\&A, 391, 369 
Kupfer T. et al., 2018, MNRAS, 480, 302

Levenhagen R. S., Diaz M. P., Coelho P. R. T., Hubeny I., 2017, ApJS, 231, 1

Marsh T. R., Nelemans G., Steeghs D., 2004, MNRAS, 350, 113

Masci F. J. et al., 2018, PASP, 131, 018003

Maxted P., 2016, A\&A, 591, A111

Morgan J. S., Kaiser N., Moreau V., Anderson D., Burgett W., 2012, in Larry M. S., Roberto G., Helen J. H., eds, Proc. SPIE Conf. Ser. Vol. 8444, Ground-Based and Airborne Telescopes IV. SPIE, Bellingham, p. OH Oke J. B. et al., 1995, PASP, 107, 375

Panei J. A., Althaus L. G., Benvenuto O. G., 2000, A\&A, 353, 970
Roelofs G. H. A., Rau A., Marsh T. R., Steeghs D., Groot P. J., Nelemans G., 2010, ApJ, 711, L138

Shen K. J., 2015, ApJ, 805, L6

Skowron D. M. et al., 2019, Science, 365, 478

Soszyński I. et al., 2013, Acta Astron., 63, 21

Terashita V., Matsushima S., 1966, ApJS, 13, 461

Tonry J. L. et al., 2018, PASP, 130, 064505

Tremblay P.-E., Bergeron P., Gianninas A., 2011, ApJ, 730, 128

This paper has been typeset from a $\mathrm{T}_{\mathrm{E}} \mathrm{X} / \mathrm{L} \mathrm{AT} \mathrm{E} \mathrm{X}$ file prepared by the author. 